\title{
Health Care Consumer's Perception of the Electronic Medical Record (EMR) System within a Referral Hospital in Kigali, Rwanda
}

\author{
Peace Uwambaye ${ }^{1^{*}}$, Kato Njunwa ${ }^{3}$, Assouman Nuhu ${ }^{2}$, Anne Kumurenzi ${ }^{2}$, Moses Isyagi ${ }^{1}$, Julienne \\ Murererehe ${ }^{1}$, Donart Ngarambe ${ }^{4}$ \\ ${ }^{1}$ School of Dentistry, University of Rwanda, College of Medicine and Health Science \\ ${ }^{2}$ School of Health Sciences, University of Rwanda, College of Medicine and Health Sciences \\ ${ }^{3}$ Director Post Graduate Studies, Director of Research, University of Rwanda, College of Medicine and Health sciences \\ ${ }^{4}$ University of Rwanda, College of Science and Technology
}

\begin{abstract} University of Rwanda, College of Health Sciences and the hospital. of a computer-based EMR and preferred paper based records.

Keywords: Electronic Medical Records, OpenClinic and Perceptions.

\section{Introduction}

Electronic Medical Records (EMR), as a health information technology innovation, has been perceived to improve efficiency and increase the effectiveness of health care delivery(Freeman, Taylor, \& Adelman, 2009). To adopt an EMR, many hospitals are actively converting from a paper-based work environment to a paperless electronic record, thus transforming the health information management (HIM) resources (Freeman et al., 2009). The primary purpose of an EMR system is to support a highquality effective integrated health care information system, which is independent of the place and time of health care delivery through Information communication technology (Hyppönen et al.,2014).
\end{abstract}

Background: Worldwide Electronic Medical Records (EMR) when compared to a paper-based system has been proven to improve service delivering numerous health care facilities. However, no research has been described in the literature regarding the user's perception of the clinical electronic medical record (EMR) system in Rwanda. The objective of this study is to evaluate the health care consumer's perception of the Electronic Medical Record (EMR) OpenClinic regarding improving the quality of health care delivery within the hospital setting. How does the OpenClinic system affect the patients' waiting time? How complete is the information obtained from OpenClinic as compared to paper medical records, and the level of satisfaction of the users of OpenClinic.

Method: A structured questionnaire was developed, validated and utilized in this quantitative research project. Quantitative data were collected from 170 participants while an interview guide was used to collect qualitative data from 6 key informants at a referral hospital in Kigali. The SPSS version 20.0 was used to analyze the quantitative data. The interviews transcribed verbatim; the data were thematically analyzed and categorized by pre-determined themes. Permission to conduct the study was approved from the ethics committee of the

Results: The majority of the participants were satisfied with OpenClinic, (90\%), while only a few (10\%) did not perceive any advantage

Conclusion: Openclinc EMR was seen to be an improvement in the data collection involving health care delivery in Kigali, Rwanda.

The introduction of EMR in the primary health care delivery system has enhanced care quality, increased safety, improved efficiency, and augmented the capacity to conduct research.(Hyppönen et al., 2014; Jha et al., 2009) Universality of an EMR allows ease in terms of compliance for chart requests, improves external and intra-office communication, and ensures a higher quality of documentation (Hyppönen et al., 2014).The EMR provides built-in protocols, improves medication management, increases comprehensive patient education, and brings together compilation of automated reports and data analysis, which does improve health care delivery. (Bates, 2002, Job, et, 2013)The EMR allows "greater and more seamless flow of information within a digital health care infrastructure, created by electronic health records (EHRs), encompasses and leverages digital progress, and can transform the way care is delivered and compensated" (Health IT.Gov.rw, 2017).

Within Africa, 15 resource poor countries have implemented an EMR. Initially instituted as a pilot program to a more comprehensive implementation phase, findings demonstrated considerable advantages over a paper based system. These advantages included enhancement of healthcare delivery, facilitation of decision-making process, increased accuracy of medication lists, legible notes and prescriptions and immediately available charts (Williams \& Boren, 2008).

The Rwandan Ministry of Health introduced an EMR system known as "OpenClinic" and "OpenMRS" in many public health facilities (Gakuba, 2009) These EMR systems are intended to support the country's vision enhanced Information Technology communication (ICT)in health care delivery. The new EMR will provide a highly effective, reliable, secure, and innovative information system supporting clinical management decisions at the bedside level as defined by the Health Sector Strategic Plan.(No-111, 2012; HSSP111, 2012). The Rwandan health system has reached a pivotal stage of development and implementation of an EMR systems to increase utilization countrywide (Health Sector Strategic plan 3; HSSP111, 2012

OpenClinic EMR developed in 1990, and has been utilized in 20 hospitals and medical clinics in Rwanda since 2007. OpenClinic,a fully-integrated hospital information system, replacing all paper based systems and archiving of patient records. Openclinic is based on a multi-tiered network design and the software is capable of servicing several thousand patients. The OpenClinic software system covers numerous different aspects of care delivery and planning within hospitals. Areas supported include patient admissions, medical records, medication administration, clinical data repository, statistical analysis, test results, pharmacy and hospital supply management, order communication, and financial manage- 
ment records (Morrison, losif, \& Danka, 2010)

Since the implementation of the OpenClinic EMR seven years ago in Rwanda there has not been any evaluation of the end users' perception and satisfaction in using this EMR. This research project collected and analyzed personal views from staffs working with OpenClinic in a referral hospital in order to examine their perceptions and evaluate satisfaction with the Openclinic.

\section{Methods}

This research project is across sectional quantitative and qualitative study at Kigali University Teaching Hospital, a referral hospital $(\mathrm{RH})$, in Kigali, Rwanda. As the pioneer in the use of OpenClinic EMR in Rwanda, Kigali University Teaching Hospitals the largest center of OpenClinic implementation in Africa with 210 work stations and 581 active OpenClinic users. (Verbeke et al., 2013) There were 581 OpenClinic users at the study site including nurses, doctors, cashiers, finance managers, pharmacists, lab technicians, system administrators, and statisticians.

For the quantitative part of the study, a total sample size of 237 , determined using Yamane's formula (Yamane, 1967), $\mathrm{S}=\mathrm{N} / 1+\mathrm{N}$ $(\mathrm{e})^{2}$ Where: $\mathrm{S}=$ Sample, $\mathrm{N}=$ Population, e: margin of error $(0.05)$, $S=581 / 1+581(0.05)^{2}=237$. A purposive stratified proportionate sampling of OpenClinic users was done to obtain the study sample and the sample size for each stratification of users and research subjects.

The data collection tool for this study was a structured five-part questionnaire adapted and modified from Msukwa (Msukwa, 2010). Meetings were arranged to meet with the OpenClinic users and explained to them the purpose, goals, and implementation of this research study. Written informed consent with signature was obtained from OpenClinic after these informational meetings. The questionnaires were distributed to the participants and they were given a three week time frame to complete at their leisure. Researchers hand collected the completed research questionnaires from the respondents. Quantitative data analysis was accomplished using Microsoft Excel and Statistical Package for Social Sciences (SPSS) Version 20.0 IBM. The data were analyzed using descriptive statistics and collated for presentation using tables and figures.

The qualitative research component included thorough in-depth interviews with six key informants including system administrators, finance managers, physicians, and support staff. These key were informants were selected because they were the heads of departments and system administrators who always access Openclinic. Before the initial interviews, the study design and objectives were shared with research participants, questions answered, and informed consent obtained from each participant prior to the beginning of interview process. The researchers requested and were granted permission from all study participants to audio record the interview, following which the interview process began. Transcription of each interview recording was completed at the end of each interview day. Analysis was accomplished by initially identifying the main themes. These themes were interpreted to examine the meanings of the words spoken and actions elicited. Validity of the data analysis was enhanced by continuous assessment of interview transcripts, frequently replaying of the recordings, and reviewing thoroughly the field notes. Commonly, the researchers utilized debriefing sessions after the interview process to help increase validity and continuity. All interviews were conducted from their work places and were in English.

Study participants were allowed to withdraw at any time during the interview process without fear of consequences, as their participation in this study was entirely voluntary in nature. The identity of the participants was kept anonymous and the records were securely stored in locked storage cabinets to ensure confidentiality.

\section{RESULTS}

A total of 230 questionnaires were distributed with a response rate of $170(74 \%)$ questionnaires were completed.

\section{Demographic Characteristics}

The minimum age of the participant was 21 years of age while the maximum was 56 years of age and the mean age was 38.5 years. The majority of the participants $131(77.1 \%)$ were age 40 years or younger, females accounting for 124 (72.9\%) () and males $46(27.1 \%)$ (). Nursing represented the largest percentage of respondents at 137 (80.6\%) compared to all other professionals like lab technicians, pharmacists and medical doctors. Most nursing respondents' educational level was advanced diploma or A1, and the secondary school level certificate (A2) which accounted for 87.8 (64.1\%). 49.2 (35\%) of the nursing respondents had Bachelors or Masters Degrees in nursing. 119 (70\%) participants had more than two years of working experience. See Table 1 for a summary of the demographic information of the study.

Table 1: Participants' demographic information $(n=170)$

\begin{tabular}{llcc}
\hline Variables & Characteristics & Frequency & Percentage \\
\hline Age (years) & $<40$ & 131 & 77.1 \\
& $40+$ & 39 & 22.9 \\
Gender & Male & 46 & 27.1 \\
& Female & 124 & 72.9 \\
Work position/ & Nurses & 137 & 80.6 \\
Qualification & Others & 33 & 19.4 \\
Education Level & A1 and A2 & 109 & 64.1 \\
& Bachelors and Masters & 61 & 35.9 \\
\multirow{2}{*}{ Working Experience } & Less or equal to 24 Months & 51 & 30 \\
& Above 24 Months & 119 & 70 \\
\hline
\end{tabular}

\section{Perception of the efficiency of the OpenClinic Electronic Medical Record}

Common themes identified in the study evaluating the users' perceptions of the OpenClinic EMR included the speed of results, accuracy of records, which increased patient safety, timeliness of results and correspondence, ease of availability, and completeness of the patient information within the system. The findings of this study show a positive perception of the OpenClinic EMR by the study participants as compared to using a paper based medical record. $111(84.7 \%)$ of the study participants were less than 40 years of age. The respondents viewed OpenClinic as fast and easy to use $105(80.1 \%), 114(87 \%)$ felt the EMR was more accurate and $99(75.6 \%)$ found the EMR information more complete. OpenClinic EMR was preferred over paper charting by 116 (88.5\%) respondents . $113(86.3 \%)$ respondents felt the EMR was overall 
safer as compared to a paper based medical record system. Among participants of less than 2 years of experience, the perception of OpeClinic EMR was positive experience as shown in Table 2,

Table 2: Perception of the participants towards the efficiency of electronic versus paper based recording

\begin{tabular}{|c|c|c|c|c|c|c|c|c|c|c|c|}
\hline \multirow[t]{4}{*}{ Perceptions } & \multirow[t]{4}{*}{ Records } & \multicolumn{2}{|c|}{ Age } & \multicolumn{2}{|c|}{ Gender } & \multicolumn{2}{|c|}{ Work Position } & \multicolumn{2}{|c|}{ Education level } & \multicolumn{2}{|c|}{ Working experience } \\
\hline & & $\begin{array}{c}\leq 40 \text { years } \\
\text { old }\end{array}$ & $>40$ years old & Male & Female & Nurses & Others & $\begin{array}{c}\mathrm{A} 1 \text { and } \\
\text { A2 }\end{array}$ & $\begin{array}{c}\text { Bachelors } \\
\text { and }\end{array}$ & $\begin{array}{c}\leq 24 \\
\text { months }\end{array}$ & $>24$ months \\
\hline & & & & & & & & & Masters & & \\
\hline & & $\mathrm{N}(\%)$ & $\mathrm{N}(\%)$ & $\mathrm{N}(\%)$ & $\mathrm{N}(\%)$ & $\mathrm{N}(\%)$ & $\mathrm{N}(\%)$ & $\mathrm{N}(\%)$ & $\mathrm{N}(\%)$ & $\mathrm{N}(\%)$ & $\mathrm{N}(\%)$ \\
\hline & Open clinic & $111(84.7)$ & 27(69.2) & 42(91.3) & $96(77.4)$ & $108(78.8)$ & $30(90.1)$ & $85(78)$ & $53(86.9)$ & $45(88.2)$ & $93(78.2)$ \\
\hline \multirow[t]{3}{*}{ Faster } & Paper form & $20(15.3)$ & 11(28.2) & $4(8.7)$ & $27(21.8)$ & $28(20.4)$ & $3(9.1)$ & $23(21.1)$ & $8(13.1)$ & $6(11.8)$ & $25(21)$ \\
\hline & Both & $0(0)$ & $1(2.6)$ & $0(0)$ & $1(0.8)$ & $1(0.7)$ & $0(0)$ & $1(0.9)$ & $0(0)$ & $0(0)$ & $1(0.8)$ \\
\hline & Open clinic & $105(80.1)$ & $25(64.1)$ & 42(91.3) & $88(71)$ & $100(73)$ & $30(90.1)$ & $77(70.6)$ & $53(48.6)$ & $43(84.3)$ & $87(73.1)$ \\
\hline \multirow[t]{3}{*}{ Easier } & Paper form & $25(19.1)$ & 13(33.3) & $4(8.7)$ & $34(27.4)$ & $35(25.5)$ & $3(9.1)$ & $30(27.5)$ & $8(13.1)$ & $8(15.7)$ & $30(25.2)$ \\
\hline & Both & $1(0.8)$ & $1(2.6)$ & $0(0)$ & $2(1.6)$ & $2(1.5)$ & $0(0)$ & $2(1.8)$ & $0(0)$ & $0(0)$ & $2(1.7)$ \\
\hline & Open clinic & $99(75.6)$ & $25(65.1)$ & $33(71.7)$ & $91(73.4)$ & 101(73.7) & $23(69.7)$ & $81(74.3)$ & $43(70.1)$ & $44(86.3)$ & $80(67.2)$ \\
\hline \multirow[t]{3}{*}{ Completeness } & Paper form & $22(16.8)$ & 12(30.8) & $6(13.0)$ & $28(22.6)$ & $30(21.9)$ & $4(12.1)$ & $23(21.1)$ & $11(18)$ & $7(13.7)$ & $27(22.7)$ \\
\hline & Both & $10(7.6)$ & $2(5.1)$ & $7(15.2)$ & $5(4)$ & $6(4.4)$ & $6(18.2)$ & $5(4.6)$ & $7(11.5)$ & $0(0)$ & $12(10.1)$ \\
\hline & Open clinic & $114(87)$ & 27(69.2) & $39(84.8)$ & $102(82.6)$ & $116(84.7)$ & $25(75.8)$ & $92(84.4)$ & $49(80.3)$ & $50(98)$ & $91(76.5)$ \\
\hline \multirow[t]{2}{*}{ Accuracy } & Paper form & $11(8.4)$ & 10(25.6) & $3(6.5)$ & $18(14.5)$ & $17(12.4)$ & $4(12.1)$ & $13(11.9)$ & $8(13.1)$ & $1(2)$ & $20(16.8)$ \\
\hline & Both & $6(4.9)$ & $2(5.1)$ & $4(8.7)$ & $4(3.2)$ & $4(2.9)$ & $4(12.1)$ & $4(3.7)$ & $4(6.6)$ & $0(0)$ & $8(6.7)$ \\
\hline
\end{tabular}

The qualitative results showed that the majority of the participants expressed positive experiences regarding the efficiency of the OpenClinic EMR. All participants reported that OpenClinic EMR improved work performance. For example "With the OpenClinic EMR we do not have to go into the filing cabinet to look

for a patient's files which take long time to find; now we just scan

in the computer and all the information about the patient appears on the

screen."P4

"We can now receive more patients in a short period of time."(P1)

\section{Perceptions of the participants towards the quality of care using OpenClinic EMR}

The findings of the study in table 3 indicate that 150 (88.2\%) of participants perceived that the quality of care had improved since the introduction of OpenClinic EMR; while 7 (4.1\%) indicated that they perceived no change in the quality of care with the EMR. The majority of the participants, $137(80.6 \%)$ perceived that the OpenClinic EMR had significantly changed the process of patient care in a positive manner. Like reduction of patients' waiting time, improving the accuracy of records, speeding up the work and also improving the safety and security of medical records.
Table 3: Perception of the participants towards the quality of care of openclinic use $(n=170)$

\begin{tabular}{llcc}
\hline & & \multicolumn{2}{c}{ Openclinic uses } \\
& & Frequency & Percentage \\
\hline Extent to which open clinic has & Significantly & 137 & 80.6 \\
changed the process of patient care & Small degree & 19 & 11.2 \\
& Not at all & 14 & 8.2 \\
Extent to which open clinic has & Improved & 150 & 88.2 \\
changed the quality of care & Not changed & 7 & 4.1 \\
& Decreased & 13 & 7.6 \\
\hline
\end{tabular}

The researchers further evaluated respondents' perceptions related to the quality of care in the in-depth interviews, most of the participants reported that there was improvement in quality of care after the introduction of OpenClinic EMR.

"It reduced patient waiting time." (P 1)

"There is no more loss of patients' documents." (P4)

"There was reduction of errors in billing." (P6)

Satisfaction of the participants with the use of OpenClinic EMR According to the type of records preferred by the participants, $136(81.9 \%)$ reported preference for the OpenClinic EMR. Nurses and other professionals reported that they were satisfied with the OpenClinic EMR in many areas of patient care including patients' medical records. Throughout the results the positive perceptions of the OpenClinic EMR was observed according to age, working experience, education level, and gender. Table 4 illustrates satisfaction scores of the participants comparing OpenClinic EMR with paper based record. 
Table 4. Satisfaction with the use of open clinic

\begin{tabular}{|c|c|c|c|c|}
\hline & & $\begin{array}{c}\text { Satisfied } \\
\mathrm{N}(\%)\end{array}$ & $\begin{array}{c}\text { Not satisfied } \\
\text { N (\%) }\end{array}$ & Total \\
\hline \multirow[t]{2}{*}{ Type of records preferred } & Open clinic & $136(81.9)$ & $30(18.1)$ & 166 \\
\hline & Paper based & $1(25)$ & $3(75)$ & 4 \\
\hline \multirow[t]{2}{*}{ Work position } & Nurses & $109(79.6)$ & $28(20.4)$ & 137 \\
\hline & Others & $28(84.8)$ & $5(15.2)$ & 33 \\
\hline \multirow[t]{2}{*}{ Age } & $<40$ & $110(84)$ & $21(16)$ & 131 \\
\hline & $40>$ & $27(69.2)$ & $12(30.8)$ & 39 \\
\hline \multirow[t]{2}{*}{ Working Experience } & $\leq 24$ months & $44(86.3)$ & $7(13.7)$ & 51 \\
\hline & $>24$ months & $93(80.7)$ & $26(21.8)$ & 119 \\
\hline \multirow[t]{2}{*}{ Gender } & Male & $39(31.5)$ & $85(68.5)$ & 124 \\
\hline & Female & $7(15.2)$ & $39(84.8)$ & 46 \\
\hline \multirow[t]{2}{*}{ Education level } & $\mathrm{A} 1$ and $\mathrm{A} 2$ & $89(74.8)$ & $20(16.8)$ & 119 \\
\hline & Bachelors and Masters & $48(94.1)$ & $13(25.5)$ & 51 \\
\hline
\end{tabular}

Participants were further asked to comment on how much they were satisfied with OpenClinic EMR in the interviews.

"I am satisfied with data processing and reporting because it's now easy and is being done in a short period of time." (P5)

"I found it more interesting."(P1)

"it did facilitate my work." (P3)

\section{Discussion}

\section{Demographic characteristics}

Research results showed the majority of the participants 131 (77.1\%) were 40 years old or less, and 39 (22.9\%) were 40 and above. Literature shows older people have less positive attitudes and a greater anxiety than their young counterparts towards EMRs and show more resistance to newer technologies than the young people (Cutler, Hendricks, \& Guyer, 2003). In the current study, participants who were aged 40 years and less, 20(15.3\%)preferred a paper based medical record compared to those who were above $40(28.2 \%)$ preferred paper based.

\section{Perception of the participants towards the efficiency of the} OpenClinic EMR versus paper based system of recording

The introduction in 2007 of an EMR in the Rwandan health care system has increased efficiency primarily through the elimination of routine tasks, such as handling paper-based charts, searching through numerous files and papers to obtain patient's medical and drug history (Kaplan, 2001) with a focus on evaluation. The literature indicates a general consensus that clinical decision support systems are thought to have the potential to improve care. Evidence is more equivocal for guidelines and for systems to aid physicians with diagnosis. There also is general consensus that a variety of systems are little used despite demonstrated or potential benefits. In the evaluation literature, the main emphasis is on how clinical performance changes. Most studies use an experimental or randomized controlled clinical trials design (RCT.OpenClinic EMR has proven to be efficient and effective worldwide. Users' perception of the OpenClinic EMR has validated and supported the increased efficacy and accuracy of the medical records as compared to a paper based record system in Rwanda. The age of respondents showed younger participants perceived that the OpenClinic EMR was faster
$111(85 \%)$, easier to use $105(80 \%)$, more accurate $114(87 \%)$ and increased medical records were more complete 99 (76\%) with the EMR than a paper based system. The qualitative interviews with participants demonstrated perceptions that the OpenClinic EMR had in fact reduced issues of increased wait time, increased the efficiency of medical record keeping, and helped facilitate accurate billing, Responses included 'There is no more loss of patients' documents" (P4).The current study of the implementation of an EMR was also supported by (Bouamrane \& Mair, 2013)). This study reported that general practitioners' perspectives on electronic medical records systems in NHS Scotland felt their EMR system improved the record keeping This current research study of the OpenClinc EMR has not only improved the efficiency but also the quality of health care delivery with in Rwanda.

\section{Perceptions of the quality of care using the OpenClinic EMR}

The OpenClinic EMR system helps improve the quality of patient care visit documentation and data, helping free up facility storage space, improving efficiency by eliminating time searching for lost charts, for example and provides immediate, access to patient records (Martin K.B. M, sukwa, \& Martin K.B. M, 2011). OpenClinic EMR has improved the quality of care in hospitals as reported by $88 \%$ of the participants in the current study. Sixty-seven percent of the participants reported that the quality of care was significantly improved in the subset of the participants, which preferred OpenClinic EMR more than a paper based record system. The key informants further expressed positive changes seen with OpenClinic EMR in interviews in which it was reported that OpenClinic EMR reduced patients' waiting time and billing issues. "It reduced patient waiting time" (P 1) "There was reduction of errors in billing" (P6)

A study in 2003 conducted in Kenya by Rotich reported that patients spent less time waiting to see a consultant (Rotich et al., 2003). The total time per visit at the health facilities was relatively shorter in duration following the implementation of an EMR helping to increase efficacy and effectiveness The EMR has been a strong capacity building to Rwanda Medical Care and needs to continue throughout the country to support a patient, family and their caregivers through safe, timely and effective documentation. 


\section{Satisfaction of the participants with the use of OpenClinic EMR}

Participants in studies by (Raghupathi \& Kesh, 2009)the digitization of health care typically has focussed simply and solely on electronic records for patients. Most systems are relational databases with a finite number of intra-enterprise applications and are limited to in-house use by health care facilities. Very few of these systems have realized fully functional, scalable, distribution capabilities, not to mention interoperability with external systems. This short-sighted tendency to build large-scale but restrictive automated systems that ignore the interactive nature of health care has resulted in limited operational success and acceptance. That said, this trend is not irreversible. Given the right circumstances, distributed, interoperable, digital health systems could emerge rapidly, offering both intra- and inter-enterprise benefits. To succeed, the design of these so-called total digital health systems (TDHSsand (Hillestad et al., n.d.) in 2005 revealed satisfactory impressions regarding EMR systems. They stated that these systems lead to major health care savings, reduced medical errors, and improved health care delivery, warned of drug interactions, sent reminders and alerts on patients with a low Body Mass Index after having their weight entered in the $\operatorname{EMR}(($ Raghupathi \& Kesh, 2009)the digitization of health care typically has focussed simply and solely on electronic records for patients. Most systems are relational databases with a finite number of intra-enterprise applications and are limited to in-house use by health care facilities. Very few of these systems have realized fully functional, scalable, distribution capabilities, not to mention interoperability with external systems. This short-sighted tendency to build large-scale but restrictive automated systems that ignore the interactive nature of health care has resulted in limited operational success and acceptance. That said, this trend is not irreversible. Given the right circumstances, distributed, interoperable, digital health systems could emerge rapidly, offering both intra- and inter-enterprise benefits. To succeed, the design of these so-called total digital health systems (TDHSs; (Hillestad et al., n.d.)2005).

Similarly to this current research study most of the participants $(80 \%)$ were satisfied with the use of OpenClinic EMR and this positive perception was further emphasized in the interviews where participants stated that this system facilitated increased efficiency in their workplace "We can now receive more patients in a short period of time'P1; "Data processing and reporting is now easy and is being done in a short period of time" P5

Freeman and colleagues in a patient and user satisfaction survey conducted in a headache specialty clinic reported that health care computerization promoted numerous positive benefits. The EMR saved time, increased accuracy, enhanced the flow of information, improved the quality of clinical data available, and reduces paperwork (Freeman et al., 2009).

\section{CONCLUSION}

This study evaluated the end user's perception of the OpenClinic Electronic Medical Records in improving the health care delivery in a referral hospital in Kigali Rwanda. Findings in the majority of users showed satisfaction with the OpenClinic EMR. This research study also supported the fact that users felt the quality of care had significantly improved with the introduction of an EMR system, and patient waiting times at the hospital had been greatly reduced. This study supported the fact the OpenClinic EMR was faster and much easier to complete, accurate, and safe compared to the use of paper based medical records and this EMR has been proven to save time and helps providers to concentrate on the needs of 'real' physical care to the patient. In general most of the participants prefer using the OpenClinic electronic medical records compared to paper based records.

\section{Acknowledgement}

We acknowledge all the participants of this study.

We are incredibly grateful to Mrs. Rebecca WHITE, Mr. Malachie TUYIZERE and Prof. Mohammed RAZZAQUE for critically reading the manuscript and providing helpful suggestions.

\section{Conflict of Interest}

None

\section{Authors Contributions}

$\mathrm{P} \cup$ contributed to the original study, data collection, analysis and manuscript writing.

$\mathrm{K} \mathrm{N} \mathrm{J} \mathrm{reviewed} \mathrm{and} \mathrm{edited} \mathrm{the} \mathrm{manuscript.}$

A N performed the quantitative data analyses for the manuscript.

A K contributed to the qualitative data analysis for the manuscript $\mathrm{M} \mathrm{I}$ and $\mathrm{J} \mathrm{M}$ contributed to the draft of the manuscript.

D N supervised the research study.

\section{References}

Bouamrane, M.-M., \& Mair, F. S. (2013). A study of general practitioners' perspectives on electronic medical records systems in NHSScotland. BMC Medical Informatics and Decision Making, 13(1), 58. http://doi.org/10.1186/14726947-13-58

Cutler, S. J., Hendricks, J., \& Guyer, A. (2003). Age differences in home computer availability and use. The Journals of Gerontology. Series B, Psychological Sciences and Social Sciences, 58(5), S271-280.

Freeman, M. C., Taylor, A. P., \& Adelman, J. U. (2009). Electronic medical record system in a headache specialty practice: a patient satisfaction survey. Headache, 49(2), 212-215. http://doi.org/10.1111/j.1526-4610.2007.01009.x

Gakuba, R. (2009). THE NATIONAL E-HEALTH STRATEGIC PLAN 2009-2013. Ministry of Health, Rwanda.

Hillestad, R., Bigelow, J., Bower, A., Girosi, F., Meili, R., Scoville, R., \& Taylor, R. (n.d.). Can electronic medical record systems transform health care? Potential health benefits, savings, and costs. Health Affairs (Project Hope), 24(5), 1103-17. http://doi.org/10.1377/hlthaff.24.5.1103

Hyppönen, H., Saranto, K., Vuokko, R., Mäkelä-Bengs, P., Doupi, P., Lindqvist, M., \& Mäkelä, M. (2014). Impacts of structuring the electronic health record: A systematic review protocol and results of previous reviews. International Journal of Medical Informatics, 83(3), 159-169. http://doi. org/10.1016/j.jjmedinf.2013.11.006

Jha, A. K., DesRoches, C. M., Campbell, E. G., Donelan, K., Rao, S. R., Ferris, T. G., ... Blumenthal, D. (2009). Use of electronic health records in US hospitals. New England Journal of Medicine, 360(16), 1628-1638. 
Kaplan, B. (2001). Evaluating informatics applications--clinical decision support systems literature review. International Journal of Medical Informatics, 64(1), 15-37.

Martin K.B. M, sukwa, \& Martin K.B. M. (2011, August). U ser Perceptions on Electronic Medical Record System ( EMR.

Morrison, C., losif, A., \& Danka, M. (2010). Report on existing open-source electronic medical records (No. 768). Cambridge CB3 OFD United Kingdom.

Msukwa, M. K. B. (2010). User Perceptions on Electronic Medical Record System (EMR) in Malawi. UNIVERSITY OF MALAWI College of Medicine, Malawi.

Raghupathi, W., \& Kesh, S. (2009). Designing electronic health records versus total digital health systems: A systemic analysis. Systems Research and Behavioral Science, 26(1), 63-79. http://doi.org/10.1002/sres.918

Rotich, J. K., Hannan, T. J., Smith, F. E., Bii, J., Odero, W. W., Vu, N., Tierney, W. M. (2003). Installing and Implementing a Computer-based Patient Record System in Sub-Saharan Africa: The Mosoriot Medical Record System. Journal of the American Medical Informatics Association : JAMIA, 10(4), 295-303. http://doi.org/10.1197/jamia.M1301

Williams, F., \& Boren, S. A. (2008). The role of the electronic medical record (EMR) in care delivery development in developing countries: a systematic review. Informatics in Primary Care, 16(2), 139-145.

Yamane, T. (1967). Statistics: An Introductory Analysis (2nd ed.). New York: Harper and Row. 